\title{
Mentalidades medioambientales: los discursos sobre el medio ambiente de los andaluces residentes en zonas urbanas
}

\author{
Jorge Ruiz Ruiz
}

Instituto de Estudios Sociales Avanzados de Andalucía (IESA-CSIC)

jruiz@iesaa.csic.es

\section{Resumen}

La conciencia medioambiental se ha extendido y generalizado socialmente en los últimos años. Sin embargo, este mayor conocimiento y sensibilización hacia los problemas del medio ambiente no se ha traducido en una extensión similar de los comportamientos medioambientalmente responsables. Esta discordancia entre conciencia y comportamiento medioambiental, puesta de manifiesto en distintas investigaciones, resulta desconcertante, pero puede ser explicada desde las mentalidades medioambientales, entendidas como concepciones del medio ambiente producidas y distribuidas socialmente. En este artículo, se presentan los resultados de una reciente investigación en la que se exploraron las mentalidades medioambientales de los andaluces residentes en zonas urbanas, mediante el análisis de los discursos producidos en grupos de discusión.

Palabras clave: mentalidades medioambientales, análisis de discursos, percepción del medio ambiente, conciencia medioambiental.

\section{Abstract. Environmental mentalities: social discourses of environmental issues of Andalusians living in urban areas}

Environmental concerns have been spread and generalised in the last few years. Nevertheless, this larger knowledge and awareness about environmental issues have not produced a similar expansion of a sense of responsibility in personal behaviours. This gap between environmental concerns and ecological behaviours has puzzled researchers. However, this can be explained by the nature of environmental mentalities, conceptions about the environment which are socially produced and spread. This paper presents the outcomes of a recent research that has explored the environmental mentalities of Andalusian people who live in urban areas. To this end, we have analysed the social discourses produced in a series of focus-groups.

Key words: environmental mentalities, social discourse analysis, environmental perception, environmental concerns.

* Los resultados que se exponen en este artículo forman parte de un estudio realizado por IESA-CSIC para la Consejería de Medio Ambiente de la Junta de Andalucía, dentro del Ecobarómetro que periódicamente realiza este Instituto. El trabajo de campo se realizó entre el 6 y el 21 de julio de 2004. Las reuniones tuvieron lugar en las salas que la empresa F\&M tiene en Sevilla. Agradezco a mis compañeros y compañeras del IESA/CSIC, especialmente a Eduardo Moyano y a Manuel Fernández Esquinas, sus oportunas críticas y aportaciones. 


\section{Sumario}

Conciencia y mentalidades medioambientales

Características generales de los discursos sobre el medio ambiente

Razones para la adopción de comportamientos respetuosos con el medio ambiente
Costes asociados a los comportamientos respetuosos con el medio ambiente

Límites a la adopción

de comportamientos respetuosos

con el medio ambiente

Conclusiones

Bibliografía

\section{Conciencia y mentalidades medioambientales}

El estudio de los comportamientos relacionados con el medio ambiente se ha enfocado, tradicionalmente desde la sociología, como un asunto de conciencia. Así, desde los años ochenta del pasado siglo, es abundante la literatura sobre la conciencia medioambiental de la población ${ }^{1}$. Este enfoque plantea una relación directa entre el conocimiento de los problemas medioambientales, la preocupación o sensibilización hacia los mismos y el mantenimiento de pautas de comportamiento responsables con el medio ambiente. En sentido inverso, bajo este planteamiento, se consideran los comportamientos medioambientalmente irresponsables como producto de la inconsciencia, es decir, de la falta de conocimiento y de sensibilización hacia los problemas medioambientales.

No obstante, este modelo comienza a presentar importantes limitaciones ya desde mediados de la década de los noventa. En concreto, desde diversos estudios empíricos, se pone de manifiesto que la relación entre conciencia y comportamiento medioambiental no es necesariamente directa. Comienza así a hablarse de inconsistencia o discordancia ( $g a p)$ entre conciencia y comportamiento medioambientales ${ }^{2}$. No se trata de negar la incidencia del conocimiento y la preocupación medioambiental sobre el comportamiento. De lo que se trata, más bien, es de introducir en los análisis una serie de factores que inciden en el modo como los sujetos articulan este conocimiento y esta preocupación en torno al medio ambiente en sus prácticas cotidianas. Podemos hablar de una tendencia a prestar una mayor atención a los estilos de vida y a las mentalidades medioambientales como factores que inciden de manera decisiva en

1. Nos referimos a los estudios sobre environmental concern o environmental awareness, y también a los estudios sobre ambientalismo. Pueden consultarse, por ejemplo, Dunlap y Liere (1983), Jones y Dunlap (1992) y Skrentny (1993). En España, pueden verse Chuliá (1995) y Navarro (1998).

2. Como señala Brand (1997), haciendo referencia diversos estudios, fundamentalmente de Schahn y Holzer (1990) y Kuckartz (1995): «[...] el axioma clásico compartido por pedagogos, políticos y activistas es que un alto nivel de conocimiento medioambiental conduce a una alta concienciación medioambiental y, por consiguiente, a los comportamientos medioambientales respectivos. Las conclusiones empíricas, sin embargo, no sostienen esta expectativa». En España, véase por ejemplo Gómez, Noya y Paniagua (1999b). 
la adopción de pautas de comportamiento respetuosas con el medio ambiente. Los estilos de vida determinan en buena medida los costes que implica la adopción de los comportamientos respetuosos con el medio ambiente. Por mentalidades medioambientales podemos entender las interpretaciones socialmente compartidas del medio ambiente que se han adquirido, reproducido y modificado dentro de las prácticas de la vida cotidiana (Brand, 1997) ${ }^{3}$.

En este artículo, se exponen los resultados de una reciente investigación sobre las mentalidades medioambientales de los andaluces menores de cuarenta años residentes en zonas urbanas. La investigación ha estado basada en una metodología cualitativa, en concreto en la utilización de la técnica del grupo de discusión. El grupo de discusión permite, mediante la confrontación de las distintas opiniones y posicionamientos, la emergencia en los discursos producidos de las representaciones socialmente compartidas de la realidad de que se trate. En este sentido, constituye una técnica especialmente apropiada para la investigación de las mentalidades medioambientales. El supuesto básico es que las distintas mentalidades sobre el medio ambiente se plasmarán en distintos discursos dominantes dentro de los grupos ${ }^{4}$.

El análisis de los discursos sobre el medio ambiente ha sido abordado por diversos autores y ha recibido múltiples contribuciones. No obstante, en la mayoría de los casos, este análisis se ha centrado en el discurso ecologista ${ }^{5}$. Nuestro análisis se centra, por el contrario, en los discursos menos formalizados que se encuentran y que circulan en la sociedad en general. Unos y otros están íntimamente relacionados. De hecho, el discurso ecologista es uno de los discursos sociales sobre el medio ambiente y sobre los comportamientos requeridos para su conservación. Además, no es un discurso cualquiera, sino que está altamente informado y goza de especial prestigio. No obstante, aquí centramos nuestro interés en el modo cómo las personas perciben los problemas medioambientales y cómo integran en su vida cotidiana los requerimientos que, respecto de su comportamiento, implica su conservación. Estas percepciones y concepciones implícitas pueden ser exploradas a través de los discursos que mantienen sobre el medio ambiente, entendidos como cristalizaciones de unas mentalidades sobre el mismo y sobre el propio papel en su conservación.

Además, los análisis de los discursos sobre el medio ambiente suelen ser de corte cuantitativo, mientras que el análisis que aquí presentamos es, por el contrario, cualitativo ${ }^{6}$. En este sentido, es un tipo de análisis de discurso que

3. Se trata de un concepto muy cercano al de representaciones sociales (Farr y Moscovici, 1984), que, en el tema específico del medio ambiente, ha sido desarrollado en los trabajos de Brand, Poferl y Schilling (1998) y, más recientemente, Brand, Fischer y Hofmann (2003).

4. Cuando hablamos de discurso dominante, nos estamos refiriendo, desde el análisis cualitativo realizado, a aquél en torno al que se producen los consensos dentro de los grupos de discusión.

5. Véase, por ejemplo, Darier (1999), Adams y Props (ed.) (2000), Hajer (1995) y Eder (1992).

6. El análisis cualitativo del discurso que aquí adoptamos se encuentra documentado en Ibánez (1989), Alonso (1998) y, en una visión más práctica, Callejo (2001). 
encuentra similitudes con el adoptado por Dryzek (1997), aunque este autor lo centra en el discurso ecologista y en el de las administraciones públicas, y, sobre todo, con el presentado por Poferl, Schilling y Brand $(1997)^{7}$.

Se realizaron cuatro grupos de discusión con residentes en una zona urbana, Sevilla capital, y definidos según dos criterios, la edad y el nivel de estudios, en la medida en que se consideran como factores fundamentales en las diferencias en cuanto a la conciencia ambiental ${ }^{8}$. Respecto de la edad, hemos considerado dos posibilidades: jóvenes entre 18 y 24 años, por un lado, y jóvenes entre 25 y 40 años, por el otro. Respecto al nivel de estudios, hemos considerado dos situaciones: con estudios superiores, por un lado, y con un nivel de estudios inferior, por el otro. Del cruce de estos dos criterios, obtuvimos cuatro grupos que quedaron definidos del modo como se representa en el cuadro 1.

Cuadro 1. Composición de los grupos de discusión realizados.

\begin{tabular}{lll}
\hline Con estudios superiores & Sin estudios superiores \\
\hline De 25 a 40 años & $\begin{array}{l}\text { Grupo de discusión número 1: } \\
\text { jóvenes de 25 a 40 años } \\
\text { con estudios superiores. }\end{array}$ & $\begin{array}{l}\text { Grupo de discusión número 2: } \\
\text { jóves de 25 a 40 años sin } \\
\text { estudios superiores y que } \\
\text { tampoco se encuentran } \\
\text { cursándolos en la actualidad. }\end{array}$ \\
\hline
\end{tabular}

De 18 a 24 años

Grupo de discusión número 3: jóvenes de 18 a 24 años con estudios superiores, o que se encuentran cursándolos en la actualidad.
Grupo de discusión número 4: jóvenes de 18 a 24 años sin estudios superiores y que tampoco se encuentran cursándolos en la actualidad.

7. Se trata de un estudio exploratorio, centrado en el contexto alemán, en el que, mediante la interpretación cualitativa de entrevistas, se muestran cinco pautas típicas a la hora de concebir y tratar los problemas medioambientales en la vida cotidiana: la orientación ecológica como proyecto de desarrollo personal, la protección medioambiental como deber cívico, la orientación de sistema o estatista, la indiferencia hacia los problemas medioambientales y la negación de los problemas medioambientales urgentes. En España, encontramos también similitudes con los recientes trabajos, no publicados, de Santamarina (2004).

8. La hipótesis de partida fue, en este sentido, que si estos dos factores inciden decisivamente en la conciencia medioambiental, pero en menor medida en los comportamientos medioambientales, es, al menos en parte, por la incidencia de una mentalidad medioambiental compartida. Como señalamos más adelante, nuestro análisis ha revelado que, al menos al nivel de discurso y en el contexto urbano de Andalucía, no se encuentran diferencias significativas en cuanto a la mentalidad medioambiental, ni en función de la edad, ni en función del nivel de estudios. Así, más que diferentes discursos, en los grupos realizados lo que se observan son distintas versiones o modulaciones de un mismo discurso dominante. 


\section{Características generales de los discursos sobre el medio ambiente}

Entre los discursos producidos por los distintos grupos realizados, no se observan diferencias discursivas significativas. Más que de distintos discursos, podemos hablar de distintas versiones, o modulaciones, de un mismo discurso dominante. Podemos considerar que este discurso dominante está ampliamente generalizado o compartido socialmente, al menos entre los colectivos considerados en nuestra investigación, es decir, los andaluces jóvenes urbanos ${ }^{9}$.

Este discurso dominante afirma el medio ambiente como un valor apreciado de manera inequívoca. En este sentido, las conductas respetuosas con el medio ambiente son consideradas como conductas morales y cívicas y, por lo tanto, socialmente deseables. Del mismo modo, las conductas irrespetuosas son sancionadas como irresponsables e insolidarias, y los argumentos que justifican estas conductas irrespetuosas son rechazados y calificados como excusas. Así, las conductas individuales respetuosas son consideradas como relevantes para la conservación del medio ambiente: la suma o agregación de estas conductas se considera que es importante para la conservación y mejora del medio ambiente. Aunque no supongan por sí mismas una solución a los problemas medioambientales, contribuyen a ella. No obstante, se señala un doble nivel en la conservación del medio ambiente, político o público y personal, y se tiende a conceder una mayor importancia al primero.

Por otro lado, la formulación del valor medioambiental suele realizarse en términos negativos, como problema presente o amenaza futura, más que en términos positivos, como bien a disfrutar. La preocupación por el medio ambiente es expresada en todos los grupos. No obstante, es una preocupación difusa o de baja intensidad. La razón parece estar en que, aunque el medio ambiente se considere un valor importante, en el discurso de los jóvenes urbanos aparece como un valor distante. Distante en el espacio, ya que corresponde más al medio natural, o al menos al rural, que al medio urbano que les es propio, y distante en el tiempo, ya que corresponde a una amenaza más futura que actual. Además, la tendencia percibida en relación con los problemas medioambientales es ambivalente. Se percibe que los problemas medioambientales son cada vez más graves, pero, a la vez, se considera que cada vez hay una mayor información y sensibilización en torno a estos temas, de manera que se confía en la solución de los problemas en el futuro.

Además de estas características compartidas de los discursos, en todos ellos encontramos también una fuerte resistencia a reconocer el propio incumplimiento de conductas respetuosas con el medio ambiente. Siendo conductas consideradas como cívicas, su cumplimiento está revestido de un fuerte carácter moral: es hacer lo que se debe o, incluso, hacer las cosas bien. En muy pocas

9. Otros discursos sociales en relación con el medio ambiente que pudieran indicar la presencia de una mentalidad diferente, habría que buscarlos en personas de mayor edad, en los que hubiera un mayor salto generacional, o bien con una diferente experiencia relacionada con el medio ambiente derivada de su lugar de residencia (rural/urbano). 
ocasiones se reconoce que no se comportan de manera respetuosa con el medio ambiente y, cuando lo hacen, reciben la recriminación del resto de componentes del grupo, de manera que no pueden explicar sus razones, ni mucho menos defender su postura ${ }^{10}$.

\section{Razones para la adopción de comportamientos respetuosos con el medio ambiente}

En los discursos analizados, los comportamientos respetuosos con el medio ambiente aparecen, fundamentalmente, como un deber moral. Son prácticas que tienen un fuerte componente moral: se trata de hacer las cosas bien o hacer las cosas mal. De este modo, estos comportamientos se conciben como un asunto esencialmente de conciencia, pero más de una conciencia individual que de una conciencia social. La dimensión fundamental es la relación del individuo con el medio ambiente, entendida como una relación respetuosa y de no-agresión. En esta dimensión fundamental, no hay una derivación práctica de los comportamientos respetuosos: no se trata de respetar el medio ambiente para obtener un beneficio, ya sea éste social o individual, sino de hacerlo como una actitud que tiene sentido en sí misma: el medio ambiente merece nuestro respeto por sí mismo.

M: Si yo le echo la culpa a este señor de que coma tal y si él lo hace pues yo también, eso no funciona así. Es que la conciencia es individual. Yo no puedo decir, no, porque las grandes industrias... No, porque no se recicla... Yo creo que la receta... es la individualidad... Los organismos públicos nos tienen que ayudar, sí, pero si uno no pone de su eso... Es como una justificación siempre, ¿no?

(Grupo de discusión número 1. Entre 25 y 40 años, con estudios superiores)

El carácter moral asociado a las conductas respetuosas con el medio ambiente aparece claramente en las dificultades que encuentran los componentes de los grupos para reconocer lo que no hacen, en la medida en que supone reconocer que se hacen las cosas mal. Parece que el factor que influye directamen-

10. Los grupos muestran una resistencia discursiva a hablar sobre los comportamientos irrespetuosos con el medio ambiente. De hecho, sólo en los dos grupos de jóvenes sin estudios superiores se reconoce abiertamente que no se realizan comportamientos medioambientalmente responsables, en concreto que no se recicla, y sólo en algunos casos puntuales. Así, ante la insistencia del moderador en centrar el discurso en los comportamientos individuales, los grupos buscan continuamente niveles de discurso refugio, como puede ser hablar genéricamente, buscar otros culpables (gobiernos, industrias) o simplemente declararse cumplidor con el medio ambiente. En este sentido, parece que la situación de grupo no es la más propicia para que afloren, en los discursos, comportamientos socialmente mal vistos, como son, en este caso, los poco respetuosos con el medio ambiente. Sin embargo, este efecto de deseabilidad social de determinadas respuestas no parece que sea más pronunciado en el caso del grupo de discusión en relación con otras técnicas de investigación social, como puede ser la encuesta. 
te en este escaso reconocimiento de los propios incumplimientos está en que es algo mal visto socialmente. Los grupos muestran, así, una marcada resistencia, incluso, a hablar de las propias prácticas y tienden a refugiarse en niveles de discurso más genéricos o impersonales. Ante la insistencia del moderador en centrarse en lo que hacemos y lo que no hacemos, los grupos reaccionan a la defensiva y eluden el tema. Por ejemplo, preguntados por su opinión sobre la reunión, en uno de los grupos, concretamente en el de jóvenes de 18 a 24 años con estudios superiores, se formula un curioso símil de ésta con un grupo de autoayuda. La semejanza percibida está en que se interpreta que lo que se requería era el reconocimiento en público de comportamientos inadecuados. El elemento de confesión que se atribuye a estos reconocimientos públicos muestra, de una manera más clara si cabe, el carácter moral atribuido a los comportamientos respetuosos con el medio ambiente.

H: Esto parece un grupo de autoayuda. [Risas.] Hace dos años que no bebo... [parodiando una confesión de un grupo de ex alcohólicos].

$\mathrm{H}$ : Y nos tenemos que confesar. [Risas]

(Grupo de discusión número 3. Entre 18 y 24 años, con estudios superiores)

El reconocimiento en público de que no se hace lo que se debería hacer es traumático y produce resistencias. No es extraño, por tanto, que tengan tan poca presencia en los discursos producidos en un contexto de grupo de discusión. En este sentido, en otro de los grupos, concretamente en el de jóvenes de 25 a 40 años con estudios superiores, se denuncia la hipocresía social en estos temas, en el sentido de no admitir los propios incumplimientos y cifrarlos siempre en los demás. En esta alusión se detecta directamente el carácter moral de los comportamientos respetuosos con el medio ambiente: mantenerlos significa ser buenos y hacer lo correcto. O, al menos, se interpreta así.

$\mathrm{H}$ : Porque todos somos medioambientales, pero hay mucha falsedad en esta sociedad. Porque creo yo que siempre nos reunimos todos y todos somos buenos, de verdad... [Risas]

(Grupo de discusión número 1. Entre 25 y 40 años, con estudios superiores)

Junto a esta dimensión moral de los comportamientos respetuosos con el medio ambiente, encontramos otras dos motivaciones para mantenerlos: una dimensión de deber cívico y una dimensión de beneficio particular. Estas dos dimensiones son esencialmente prácticas: responden a un interés práctico, a la obtención de un resultado, de un beneficio, social uno de ellos y particular el otro. La dimensión de los comportamientos respetuosos con el medio ambiente como conductas cívicas se deriva de su faceta de contribución a un bien común: la conservación del medio ambiente. La imagen repetida en todos los grupos para referirse a esta dimensión de los comportamientos respetuosos con el medio ambiente es la del granito de arena. La idea es que, aunque los com- 
portamientos respetuosos no solucionen los problemas medioambientales, al menos contribuyen a su mejora. Pero también hay una idea de agregación, según la cual los comportamientos respetuosos adquieren sentido social cuando se consideran en su conjunto. Habría un entre todos, que presupone el cada uno de nosotros, como mecanismo de consecución de un bien social, la conservación del medio ambiente, que se considera fundamental. Considerados aisladamente, los comportamientos respetuosos pueden parecer irrelevantes, pero no cuando se consideran en su conjunto. No es fundamental que lo haga todo el mundo, ni siquiera la mayoría, aunque sería lo deseable: basta con que lo haga mucha gente y, en perspectiva, que lo haga cada vez más gente. Hay una percepción de la utilidad social del coste particular que suponen estos comportamientos y que supone centrarse en quienes lo hacen, o en quienes lo hacemos, más que en quienes no lo hacen. En este sentido, sería un comportamiento altruista y supondría un razonamiento inverso al de la ausencia de compensaciones y la ruptura de la solidaridad, que veremos más adelante como algunas de las razones negativas para mantener los comportamientos respetuosos con el medio ambiente.

$\mathrm{H}$ : Vale aquí el tópico de lo que se dice que cada persona puede hacer una cosa que tiene mucha importancia, que granito a granito, porque en otras cosas se dice y suena muy tópico, y entre comillas puede ser verdad. Pero en esto de que cada persona trate de hacerlo mejor esté dónde esté, o cuide la naturaleza o aquí mismo. Yo creo que persona a persona, lo que va haciendo tiene su importancia, mucho más que en otras cosas.

(Grupo de discusión número 3. Entre 18 y 24 años, con estudios superiores)

Las razones morales y cívicas para mantener comportamientos respetuosos con el medio ambiente están, sin duda, íntimamente relacionadas. De hecho, una de las razones por las que estos comportamientos son considerados como buenos es porque son útiles socialmente, porque contribuyen al bien común de la conservación del medio ambiente. Sin embargo, la diferencia entre unas y otras razones aparece claramente cuando se expresan dudas acerca de la utilidad de los comportamientos respetuosos. Estas dudas suelen ir asociadas a la creencia de que la mayoría no mantiene estos comportamientos. Desde el punto de vista de la utilidad para la conservación del medio ambiente, es muy importante que sean conductas generalizadas: lo que confiere sentido y utilidad a estos comportamientos es que se realicen por todos, la mayoría o, al menos, muchos ciudadanos. De esta manera, como veremos más adelante cuando abordemos el tema de las razones medioambientales negativas, el hecho de que otros no lo hagan plantea dudas sobre el beneficio para la conservación del medio ambiente que producen estos comportamientos. Pues bien, en los discursos de los grupos se afirma que los comportamientos respetuosos con el medio ambiente se deben realizar al margen de lo que hagan los demás. Siempre se contribuye en algo, es mejor hacerlo que no hacerlo. Pero, sobre todo, en este argumento se entepone la conciencia individual, lo 
que se sabe que está bien, a la utilidad social puesta en duda. Es algo que debemos hacer al margen de que sea más o menos útil.

Junto a estas razones morales y cívicas, aparece, respecto de determinados comportamientos respetuosos con el medio ambiente, una tercera motivación que podemos denominar como el interés o el beneficio particular derivado de las mismas. Incluso para alguno de estos comportamientos, estas motivaciones particulares son las que se perciben más claramente y las que tienen más fuerza. Es el caso del consumo de alimentos ecológicos respecto del que apenas se percibe el beneficio para el medio ambiente, pero que es claramente identificable como beneficioso para la salud. La motivación principal atribuida al consumo de estos alimentos sería más el beneficio particular que el beneficio social, más la calidad del producto y su carácter saludable que el haberse producido de manera respetuosa con el medio ambiente. Pero en la cuestión que más directamente aparece este interés particular quizás sea en el ahorro doméstico de electricidad y de agua. Además del beneficio medioambiental, este ahorro tendría una repercusión económica directa sobre la factura a pagar. Por supuesto, estas razones o motivaciones centradas en el interés particular no son incompatibles con las razones morales y cívicas. Normalmente, se entienden como motivaciones adicionales para hacer lo que se debe y beneficia al medio ambiente. Pero incluso en algunos discursos se afirma la primacía de este tipo de motivaciones, ahora bien, siempre referido al comportamiento de los demás y rara vez al propio, porque, como venimos diciendo, eso está mal visto.

M: Mi abuela, te aseguro que no ahorra agua por no gastar, es porque el contador luego... [Risas]

(Grupo de discusión número 4. Entre 18 y 24 años, sin estudios superiores)

\section{Costes asociados a los comportamientos respetuosos con el medio ambiente}

Las prácticas respetuosas con el medio ambiente son percibidas como costosas, en el sentido de que implican, en mayor o menor medida, asumir un coste asociado a su realización. Pero este coste no es en sí mismo un factor que inhiba su realización, sino que se asume como algo consustancial al comportamiento respetuoso con el medio ambiente. Así, un comportamiento respetuoso con el medio ambiente que no tenga coste, no es percibido como tal: sería simplemente un comportamiento que no perjudica al medio ambiente. Lo que distingue al comportamiento medioambiental es su carácter costoso para el individuo que lo adopta.

H: Lo que es calidad de vida también está enfrentado a lo que es el medio ambiente. Para conseguir un medio ambiente mejor, lo primero que habría que hacer sería una serie de sacrificios que el personal, digamos de a pie, no está dispuesto a hacer.

(Grupo de discusión número 1. Entre 25 y 40 años, con estudios superiores) 
No sólo es que la conservación del medio ambiente se traduzca en prácticas que requieren un esfuerzo. También es que determinados modos de vida característicos de la vida urbana conllevan necesariamente efectos perjudiciales para el medio ambiente. Los requerimientos para la conservación del medio ambiente se perciben, así, como esencialmente contrarios a los valores y a los principios de la sociedad de consumo. En concreto, el valor medioambiental se contrapone a la comodidad como valor propio de las sociedades de consumo $y$, en particular, de la vida en las ciudades. Esta contradicción entre valores dominantes se resuelve en buena medida, como veremos más adelante, relativizando el esfuerzo o la renuncia a realizar por razones medioambientales. No obstante, no está bien visto, no es defendible en público, que se dejen de realizar prácticas importantes para el medio ambiente por comodidad. Por ello, casi siempre que aparecen en los grupos este tipo de argumentos, se hace en términos impersonales o bien refiriéndolos a otros, a los demás.

Las exigencias que implican los comportamientos respetuosos con el medio ambiente son percibidas con frecuencia como incompatibles con la vida moderna, en general, y con la vida en las ciudades, en particular. Los modos de vida que les vienen impuestos, o al menos que se perciben como ineludibles, implicarían necesariamente comportamientos contaminantes o despilfarro de energía, por ejemplo. El margen de decisión percibido es, en este sentido, muy limitado. Y, en cualquier caso, la adopción de los comportamientos respetuosos con el medio ambiente implica siempre un mayor o menor coste que hay que asumir para mantenerlos e incorporarlos a los hábitos cotidianos. La aplicación estricta de lo que hay que hacer, o al menos de lo que ellos consideran que podrían hacer, se percibe como irrealizable, en la medida en que conllevaría un coste inasumible. La realización al menos de manera limitada aparece así en los grupos como un modo de lavar una conciencia que está, necesariamente, manchada.

$\mathrm{H}$ : Es que un continuo sin-vivir, es decir, o soy ecologista, o soy urbano. O soy el urbano éste que lava su conciencia, cogiendo sus plastiquitos llevándolos a lo del tema del plastiquito, los papeles al de los papelitos y eso que ni siquiera sabemos, lo que estábamos antes hablando, si es verdad si sirve para algo, pero por lo menos nos limpia la conciencia.

(Grupo de discusión número 1. Entre 25 y 40 años, con estudios superiores)

En los grupos, aparecen con frecuencia argumentos que consideran los requerimientos y las exigencias medioambientales como exagerados. Por un lado, estas exigencias se refieren a casi todos los aspectos de la vida, principal aunque no exclusivamente al ámbito doméstico. Pero también es que realizar bien las exigencias medioambientales requiere de un grado de excelencia muy elevado. El resultado es una sensación de que, por mucho que se haga, siempre se dejan cosas por hacer o no se terminan de hacer adecuadamente. Esta incapacidad asumida para responder de manera suficiente a las exigencias de la conservación del medio ambiente se traduce, como veremos más adelante, en 
un sentimiento de culpa y lleva a una relativización de las mismas. Aquí nos interesa resaltar esta percepción de un excesivo y creciente coste de los comportamientos medioambientales.

$\mathrm{H}$ : Con el plástico te dicen hasta que cortes las botellas antes de meterlas... M: Anda, no me digas.

H: Sí, sí. De todas formas, dicen que eso también puede ser medio leyenda, que en Alemania... [Risas.] Tú la lata de tomate, cogen el metal lo limpian, porque sucio tampoco es del todo limpio, quitan el papelito, entonces el papel al tema del papel y el metal al tema del metal... Y así con todo...

(Grupo de discusión número 1. Entre 25 y 40 años, con estudios superiores)

Podemos distinguir, dentro del discurso de los grupos, cuatro tipos fundamentales de costes que implican los comportamientos respetuosos con el medio ambiente: comportamientos que suponen una molestia, comportamientos que implican un esfuerzo, comportamientos que conllevan una renuncia y comportamientos que tienen un coste económico. Por ejemplo, el reciclaje implicaría una molestia, almacenar en varias bolsas, y un esfuerzo, llevar las distintas bolsas a los distintos puntos dispuestos para su depósito. La limitación del uso del automóvil es el caso prototípico de comportamiento que implica una renuncia, generalmente a la comodidad que supone el uso del coche particular, pero también al estatus asociado al mismo, aunque también implica una molestia, depender del transporte público, o un esfuerzo, desplazarse en bicicleta o andando. La limitación del uso del aire acondicionado, de otros electrodomésticos, o del uso doméstico del agua serían otros comportamientos que implican renuncia a la comodidad asociada a su uso. Por último, entre los comportamientos que suponen un coste económico, encontramos, por ejemplo, aquéllos asociados a muchas decisiones de consumo, sobre todo de los alimentos ecológicos, o de instalación de mecanismos para el uso doméstico de energías renovables, sobre todo la energía solar.

El coste que se asume es, en la mayoría de los casos, limitado y viene determinado por la valoración que del mismo haga el individuo, que a su vez está estrechamente relacionado con las condiciones de vida particulares de cada uno. Por ejemplo, no es lo mismo separar las basuras en una casa amplia que admite la organización del espacio que en una casa con el espacio reducido. Así, el coste no viene determinado sólo por la práctica de que se trate en cada caso, sino también por los condicionantes que encuentra el individuo para su realización.

M: Yo como lo tiro todo en una bolsa... No puedo poner una bolsa encima de la encimera porque me falta tiempo y me falta espacio para poner una bolsa para eso. Que reconozco que está mal hecho, pero...

(Grupo de discusión número 2. Entre 25 y 40 años, sin estudios superiores)

Una de las quejas más frecuentes que se formula en los grupos se refieren a la falta de ayudas para la realización de comportamientos respetuosos con el 
medio ambiente. Se demanda, en este sentido, que se dispongan más medios que faciliten estos comportamientos, como un modo de reducir los costes que para el ciudadano lleva asociada su realización. La disposición de mayor número de depósitos de basuras es una de las demandas más habituales en este sentido, si bien se formulan otras demandas, como, por ejemplo, el abaratamiento de los alimentos ecológicos.

M: O para tenerte que ir tres calles más para abajo. [Risas.] Porque, vamos, yo parezco tonta todas las mañanas, andando medio kilómetro para ir a tirar una botella de cristal o un frasquito de aceitunas. No hay infraestructura para que te conciencies.

(Grupo de discusión número 1. Entre 25 y 40 años, con estudios superiores)

\section{Límites a la adopción de comportamientos respetuosos con el medio ambiente}

En los discursos de los andaluces residentes en zonas urbanas aparecen cuatro razones fundamentales para no adoptar, total o parcialmente, conductas respetuosas con el medio ambiente: el desconocimiento, la desconfianza, la relativización de las exigencias y la ausencia de compensaciones. En este apartado vamos a referirnos a cada una de ellas por separado, pero siempre teniendo en cuenta que no son incompatibles unas con otras.

\section{Desconocimiento}

El desconocimiento es el argumento más frecuente en los discursos de los grupos para justificar el incumplimiento de los deberes medioambientales. Pero también es el argumento más débil, en la medida en que implica una responsabilidad personal: si se desconoce algo es, al menos en parte, por desinterés o despreocupación. La primera justificación para dejar de hacer algo que se considera que se debe hacer es el desconocer, bien qué es lo que hay que hacer, o bien cómo se hace. Por supuesto, este argumento se refiere sobre todo a comportamientos complejos o que requieren conocimientos muy específicos. No obstante, también lo encontramos en comportamientos aparentemente más sencillos, como puede ser lo que se identifica en los grupos como reciclaje, es decir, el almacenaje y depósito selectivo de residuos domésticos.

M: Yo reciclaba..., hace un tiempo ya que no lo hago, pero antes sí hacía lo de las botellas estas de vidrio y eso, las apartaba y cuando pasaba un cierto tiempo, que tenía ocho o nueve botellas, cogía y las llevaba. Pero ya de un tiempo a esta parte la verdad es que no, no lo vengo haciendo. Porque entre que todavía no me he enterado de lo de los cubitos, cuál es, de los nuevos estos, no sé cuál es, si es el amarillo, no tengo ni idea y para meterlo en otro que no... Prefiero saber antes en cuál es.

(Grupo de discusión número 4. Entre 18 y 24 años, sin estudios superiores) 
La complejidad percibida de los comportamientos requeridos es, no obstante, el principal factor que incide en el desconocimiento. Así, rara vez se argumenta que se desconoce el requerimiento en sí, sino más bien las implicaciones que este comportamiento tiene o el modo como se realiza correctamente. En este sentido, como se comentaba antes, se percibe que hay una complicación progresiva de los comportamientos requeridos. En los grupos, formados por personas relativamente jóvenes, se percibe esta complicación referida a ellos mismos, pero se considera que es mucho más grave para las personas mayores, en la medida en que no han incorporado en su educación la información y los conocimientos que permiten una comprensión adecuada de los comportamientos requeridos.

La incomprensión llevaría, incluso, a desconfiar de la utilidad misma del comportamiento requerido, lo que muestra una conexión entre desconocimiento y desconfianza: se desconfía de la utilidad, en buena medida, porque se desconocen y, en consecuencia, no se comprenden los procesos de los que los comportamientos requeridos son una parte. La mayoría de los desconocimientos argumentados están referidos al depósito de materiales peligrosos, como pueden ser pilas, el mercurio de un termómetro o el aceite doméstico. El tema del aceite como residuo doméstico es, sin duda, el que aparece más a menudo como generador de dudas en los grupos. La respuesta más frecuente es tradicional: reutilizarlo para hacer jabón. Esta solución tradicional, que se identifica como propia de las madres o de las abuelas, no se adopta como propia. Hacerlo desaparecer a través de las cañerías es la solución, que, si bien no se formula explícitamente, se deja entrever como más frecuente.

$\mathrm{M}$ : El aceite es lo que más contamina y es una cosa que por ejemplo... Eso es lo que más contamina en el mundo. Pero que tampoco hay un sitio..., por lo menos información, porque yo desconocía a dónde se puede llevar el aceite usado.

(Grupo de discusión número 4. Entre 18 y 24 años, sin estudios superiores)

En los grupos se formula una queja acerca del carácter complejo y técnico de mucha de la información que manejan sobre el medio ambiente. Esta queja es más frecuente en los grupos de jóvenes sin estudios superiores, pero, aunque no se formule directamente, en algunas de las intervenciones de los jóvenes con estudios superiores también aparecen dificultades de comprensión de los comportamientos requeridos. En particular, se percibe una excesiva complejidad en las informaciones que provienen de los grupos y de las asociaciones ecologistas. Esta apreciación refleja una brecha o un distanciamiento, al menos percibido, entre los ecologistas o activistas del medio ambiente y la población en general.

M: Las informaciones parecen que son para gente especializada. La poca información que hay, la mayoría no se entiende. Tú se la das a mi hermana con doce años, muy bonito el sol verde...

(Grupo de discusión número 4. Entre 18 y 24 años, sin estudios superiores) 
Parece que el desconocimiento al que se refieren y que argumentan los grupos se refiere a dos cuestiones diferentes, aunque íntimamente relacionadas. Por un lado, a la falta de información sobre lo que podemos hacer para mejorar el medio ambiente y sobre cómo hacerlo y, por otro lado, a la sensibilización o concienciación sobre la importancia que tiene el hacerlo. La ignorancia adquiere, por tanto, el doble sentido de no saber y de desconsiderar, restar importancia. En este sentido, existe una percepción de una creciente sensibilización que correría paralela a la mayor y mejor información disponible. Por supuesto, se considera que la sensibilización aumenta con la información, pero se señala repetidamente en los grupos que ésta no es suficiente. El acento se pone en la educación ambiental como el factor que permite al individuo adquirir una mayor concienciación, además de una mayor capacidad de comprensión de la información disponible. En este sentido, se señala en los grupos una diferencia fundamental de las generaciones más jóvenes respecto de las anteriores. Para las personas mayores, sería más difícil adoptar los comportamientos respetuosos con el medio ambiente, porque, o bien no entienden lo que se requiere de ellos, o bien no le conceden importancia.

H: Nuestros padres están menos concienciados, lo que decía ella, por ejemplo que sus padres no separan, pero que nosotros vamos a... Y aunque no lo pensemos, pero hacemos más que... lo de las aguas, todo eso. Que yo creo que estamos en ese aspecto en general más concienciados de lo que...

(Grupo de discusión número 3. Entre 18 y 24 años, con estudios superiores)

También se formula una crítica a la educación que se recibe de los padres. La escasa concienciación de las generaciones anteriores respecto de los problemas medioambientales tendría una influencia muy negativa sobre la educación que se da a los hijos en esta materia. Incluso en algunos grupos se plantea la cuestión de que son los hijos los que educan a los padres, tanto informándoles sobre temas medioambientales, como concienciándoles de la importancia de los comportamientos respetuosos con el medio ambiente. Toda esta argumentación resalta la idea de que el desconocimiento, la desinformación y la falta de sensibilización, afectándoles a ellos, a los más jóvenes, es sobre todo un problema de los mayores, de las generaciones anteriores. Sin embargo, también encontramos posicionamientos más autocríticos, que reconocen la escasa preocupación e implicación de los jóvenes respecto de los problemas medioambientales. Una vez más, la comodidad se señala como un valor alternativo a la realización de comportamientos respetuosos con el medio ambiente. Ahora bien, la comodidad no es un argumento que justifique la no-realización de estos comportamientos. Incluso referidos a los jóvenes, este tipo de argumentos se plantean en términos impersonales: es la gente, y no nosotros, la que va a su bola.

M: Yo creo que la generación de nosotros es un poco... Muy poco conscientes. Y flojos a punta pala, vamos. Porque... Pues porque la gente va a su bola y la gente va...

(Grupo de discusión número 4. Entre 18 y 24 años, sin estudios superiores) 
Además de la educación ambiental, otro factor que se considera importante para aumentar la concienciación respecto de los problemas medioambientales son las campañas informativas. En general, los grupos se muestran muy críticos con estas campañas. Por un lado, se consideran poco impactantes, lo que les restaría notoriedad en un contexto de saturación de información. El referente se establece respecto de las campañas de sensibilización contra el maltrato doméstico o para prevenir los accidentes de tráfico. Pero, sobre todo, se critica que son campañas poco relevantes, en el sentido de que no informan sobre las consecuencias futuras de no adoptar comportamientos respetuosos con el medio ambiente. Esta consideración de la relevancia del mensaje está relacionada con un distanciamiento temporal respecto a los problemas medioambientales. Se trataría, en este sentido, de acercar los problemas al presente, mediante mensajes que anuncien las consecuencias futuras.

M: Por ejemplo, en accidentes te ponen el accidente y ponen lo que te pasa después de haber tenido el accidente. En el medio ambiente no, en el medio ambiente te ponen la papelera, recicla, no sé qué, ahorra agua, esto lo otro...

M: Es lo que ha dicho ella, saber..., si tú no lo haces, lo que puede traer después. (Grupo de discusión número 4. Entre 18 y 24 años, sin estudios superiores)

\section{Desconfianza}

La desconfianza es otra de las razones que se esgrimen en los grupos para no adoptar comportamientos respetuosos con el medio ambiente. Esta desconfianza está referida, fundamentalmente, a la utilidad percibida de los comportamientos respetuosos con el medio ambiente y, por consiguiente, del coste asociado a estos comportamientos. Esta utilidad percibida se perfila así en los grupos como un factor fundamental para la adopción de los comportamientos respetuosos con el medio ambiente, en la medida en que justifican que se asuma el coste asociado a los mismos. De manera inversa, la desconfianza o las dudas sobre dicha utilidad aparece en los grupos como un argumento que fundamenta el recelo hacia los comportamientos requeridos o, incluso, el rechazo de los mismos.

$\mathrm{H}$ : Pero es que yo lo estoy haciendo, es decir, yo lo hago. Pero de lo que me doy cuenta es que por el hecho de que yo lo haga no significa en absoluto que las cosas estén mejorando ahora nada...

(Grupo de discusión número 1. Entre 25 y 40 años, con estudios superiores)

En torno a la utilidad de los comportamientos respetuosos con el medio ambiente se generan en los grupos importantes dudas, referidas a dos cuestiones fundamentales. Por un lado, a la incomprensión de la importancia o relevancia percibida de la propia contribución a la conservación del medio ambiente, lo que lleva a cuestionar el alcance o la repercusión real de dichas 
contribuciones. En este sentido, las dudas minimizan la trascendencia percibida de la realización o no de los comportamientos respetuosos con el medio ambiente. Por otro lado, se duda también de la gestión pública del resultado o producto de estos comportamientos o, dicho de otro modo, del aprovechamiento de la contribución realizada.

No, obstante, no está bien visto dudar o desconfiar. De hecho, en la mayoría de las ocasiones en las que aparece esta desconfianza en los discursos suele provocar reacciones en el grupo que descalifican estos argumentos como excusas para no asumir el coste asociado a los comportamientos medioambientalmente responsables. Ésta parece ser la razón por la que, con frecuencia, cuando se formulan estas dudas se les atribuyen a otros o se expresan en términos impersonales. Rara vez las dudas expresadas se asumen como propias, aunque, al hacerse eco de ellas, los discursos revelan que se comparten o, al menos, se les concede crédito.

M: Y bueno, una duda que queda, si luego el reciclado se recicla realmente... $\mathrm{H}$ : Eso es otra historia. A mí, un amigo me dice: «Yo cómo voy a reciclar, si yo estoy viendo que los mismos contenedores se están tirando donde se tiran las demás, porque es que no hay...".

(Grupo de discusión número 1. Entre 25 y 40 años, con estudios superiores)

La desconfianza suele ir asociada a una visión crítica del comportamiento de los otros y, más en particular, de las políticas y actuaciones de los poderes públicos en esta materia. Se advierte, en este sentido, una incomprensión de muchas de las medidas que, en materia de medio ambiente, adoptan los gobiernos. Esta incomprensión afecta directamente al sentido de los propios comportamientos respetuosos con el medio ambiente, en la medida en que limitan o incluso cuestionan su utilidad percibida. En los discursos de los grupos se refleja una fractura o desconexión percibida entre los comportamientos ciudadanos requeridos y las decisiones políticas en materia de medio ambiente. También se expresa una marcada desconfianza hacia la información que reciben sobre los problemas que afectan al medio ambiente y sobre los comportamientos vinculados a su conservación. Sobre todo, se desconfía de la información comercial: la publicidad se percibe como información interesada poco confiable. Se advierte, por ejemplo, un desgaste del término ecológico provocado por un abuso percibido en su utilización. Esta desconfianza, que en principio se refiere sobre todo a la información comercial que utilizan las empresas para publicitar sus productos, parece que también se extiende a otras informaciones y a otros ámbitos, como un efecto de contaminación informativa. La información recibida con relación a temas medioambientales se percibe, con frecuencia, como parcial e interesada.

H: Cada vez se están volcando más las empresas en lo ecológico, no sé cuántos. ¿Por qué? Porque la gente cada vez presiona más, se estudia y le dicen: 
«Oye, que la gente va por aquí». Y como lo que les interesa es seguir ganando dinero, pues lo van a hacerlo de manera que...

$\mathrm{H}$ : Lo que van a hacer es engañarte diciéndote que lo hacen y luego por detrás no lo harán.

(Grupo de discusión número 1. Entre 25 y 40 años, con estudios superiores)

La desconfianza está muy relacionada con la percepción de otros culpables, o mejor de otros más culpables, con la percepción de una responsabilización o culpabilización excesiva del ciudadano. A esta cuestión nos referiremos a continuación. Aquí nos interesa resaltar cómo la utilidad percibida de los propios comportamientos no es algo que se considere o se evalúe de manera aislada. Por el contrario, la percepción del comportamiento de los otros establece un baremo o medida de la importancia atribuida a nuestro propio comportamiento. Pero, sobre todo, es la percepción e incomprensión de lo que hacen los que tienen mayor capacidad de actuación, ya sean los gobiernos o las empresas, lo que genera mayores dudas y desconfianzas hacia la utilidad y trascendencia de los propios comportamientos.

M: Ahí se te quitan las ganas, cuando te enteras de esas cifras se te quitan las ganas, ahí realmente a mí se me quitan las ganas de ahorrar agua. Cuando me repercute, por ejemplo, en invierno que hace mucho frío y mientras me enjabono está el agua caliente, que la cierro... ¡No la cierro! Si eso no es nada para lo que despilfarra la agricultura y la industria...

(Grupo de discusión número 1. Entre 25 y 40 años, con estudios superiores)

\section{Relativización de las exigencias}

Los comportamientos respetuosos con el medio ambiente en su conjunto, incluso alguno de ellos considerado aisladamente, son percibidos como excesivamente exigentes. Tanto por extensión, por la cantidad de las conductas y las prácticas implicadas, como por intensidad, los requerimientos para realizarlas correctamente, el respeto hacia el medio ambiente se configura, en el discurso de los grupos, como un cúmulo de exigencias casi ilimitado. Esta consideración lleva, en buena medida, a la constatación de que es prácticamente imposible cumplir todos y cada uno de los deberes medioambientales: en la práctica, siempre quedan deberes que cumplir o bien no se cumplen de manera suficiente. Esto genera una sensación de culpabilidad necesaria: por mucho que se haga, siempre se podría hacer más, o bien siempre se podría hacer mejor ${ }^{11}$.

$\mathrm{H}$ : Yo tengo muchas veces la sensación de que el comportamiento que tenemos negativo es vivir. Dices: «Lo siento, lo siento, siento existir». [Risas.] Porque

11. Esta culpabilización percibida del ciudadano muestra, una vez más, el carácter moral asociado a los comportamientos respetuosos con el medio ambiente, en el sentido de que son percibidos como un modo de limpiar la conciencia ante una supuesta culpabilidad. 
es continuamente..., si te pones a reflexionar, si lo llevas en plan... Como te pongas a pensar un poquito dices: «¡Dios mío, qué culpable soy por mi coche... Por..., por..., por...!».

(Grupo de discusión número 1. Entre 25 y 40 años, con estudios superiores)

La culpabilidad es inevitable, en la medida en que deriva de la incompatibilidad percibida del respeto al medio ambiente con los modos de vida imperantes, sobre todo en las ciudades. De esta manera, se plantea la alternativa entre los valores ecologistas y los valores de la sociedad de consumo como un dilema irresoluble, que se traduce directamente en una culpabilidad ineludible. La contradicción intrínseca entre los valores de comodidad y de respeto al medio ambiente es percibida, por tanto, como una trampa de la que no es posible escapar y que trae como resultado inevitable un sentimiento de culpa, de no hacer lo que se debe hacer o, al menos, de no hacerlo suficientemente.

H: El personal digamos que ya está, ha conseguido un cierto nivel de comodidad y no está dispuesto a echar para atrás por mejorar el medio ambiente. Que realmente es lo que tú dices, el ciudadano de a pie no tiene la culpa...

(Grupo de discusión número 1. Entre 25 y 40 años, con estudios superiores)

Esta sensación de culpa no tiene por qué ser necesariamente desmotivante: de hecho, la culpa es uno de los principales mecanismos que apuntalan las normas en nuestra cultura. No obstante, esta culpa también puede generar rechazo si se percibe como excesiva. La actitud pasota o despreocupada se plantea en los discursos como un antídoto contra esta sensación de culpa inevitable. Puede parecer que lo que se plantea es un dilema entre cumplir estrictamente los requerimientos medioambientales o ignorarlos totalmente ${ }^{12}$. No obstante, lo que predominan son las soluciones intermedias. El rechazo de la culpabilización lleva con frecuencia, en este sentido, a una relativización de las exigencias percibidas. La alusión a quienes pasan totalmente puede ser entendida como un modo de compensar la culpa asociada a no cumplir de manera estricta: uno puede que no sea tan bueno como debería ser, pero tampoco es tan malo como quienes se despreocupan totalmente, como esos otros que pasan. Si me diferencio de los malos, hago más asumible mi posición intermedia.

La relativización de las exigencias de conservación del medio ambiente puede ser interpretada como una estrategia para hacer compatible unas normas que se consideran necesarias y deseables, con unas condiciones y unos modos de vida igualmente deseables y, en muchas facetas, percibidos como insoslayables. En los grupos se plantean los modos de vida contaminantes casi como una imposición, de manera que el margen de elección sería muy redu-

12. En este contexto, el término ignorar se refiere a desatender o despreocuparse, más que a desconocer. No obstante, nos interesa en este punto recalcar esta doble dimensión informativa (no se sabe) y de actitud (no se quiere saber) de los posicionamientos más despreocupados. 
cido. No es el lugar para discutir en qué medida estos modos de vida contaminantes pueden ser o no evitados por las personas, en qué medida son impuestos socialmente y en qué medida admiten la libre elección. Aquí nos limitamos a constatar que los grupos perciben que muchas de las prácticas cotidianas que deterioran el medio ambiente son inevitables. Su capacidad para vivir de otra manera es percibida como limitada, por lo que necesariamente se ven obligados a mantener prácticas poco respetuosas con el medio ambiente o, dicho de otro modo, a adoptar conductas respetuosas con el medio ambiente, pero sólo hasta un determinado punto.

M: Claro, pero le digo una cosa, las medidas que tomemos que no sean incompatibles con la vida. Porque ya ducharse es ya es una cosa...

(Grupo de discusión número 1. Entre 25 y 40 años, con estudios superiores)

El problema de esta relativización de las exigencias medioambientales es que introduce dos efectos muy peligrosos respecto de los sistemas normativos de respeto al medio ambiente: un efecto de relajación y un efecto de discrecionalidad. La relajación se refiere a bajar el nivel de exigencia que, en general, se aplica a las prácticas respetuosas con el medio ambiente: hacer todo pero en menor medida. La discrecionalidad se refiere a la selección, dentro de todo el conjunto de normas medioambientales, de aquéllas que considero que puedo realizar y aquéllas otras que no me puedo permitir: por ejemplo, sí reciclo pero no limito mi uso del coche. En cualquier caso, la relativización de los conductas respetuosas con el medio ambiente supone introducir una rebaja en las exigencias asumidas $y$, por lo tanto, introducir en las prácticas cotidianas conductas poco respetuosas con el medio ambiente, bajo una forma moralmente aceptable.

$\mathrm{H}$ : El problema está en qué está dispuesto el personal a sacrificar a cambio de lograr un medio ambiente más limpio...

(Grupo de discusión número 1. Entre 25 y 40 años, con estudios superiores)

Otra estrategia discursiva que aparece en los grupos para compensar la excesiva culpabilización percibida de los ciudadanos, es la de buscar otros culpables. La culpa se relativiza también en comparación con la responsabilidad que tienen los demás y, en particular, los otros más influyentes. Si hay otros culpables y, sobre todo, si hay otros más culpables, mi propia culpa se relativiza y se hace más asumible. Los comportamientos ciudadanos pueden ser importantes, pero más importantes y trascendentes son las normativas medioambientales y su cumplimiento. Esta forma de valorar los propios comportamientos en comparación con los que presentan los otros, nos introduce directamente en la cuarta razón para no adoptar comportamientos respetuosos con el medio ambiente, a la que hemos denominado «ausencia de compensaciones», y que se refiere a la percepción de la plasmación social de los requerimientos medioambientales como un juego descompensado. 
M: Concienciar a las empresas, los que... no sé, los que vierten, tantas cosas. Los ciudadanos también, pero hay mucha parte también, no sé, a lo mejor...

(Grupo de discusión número 3. Entre 18 y 24 años, con estudios superiores)

\section{Ausencia de compensaciones}

Los comportamientos respetuosos con el medio ambiente precisan para adquirir sentido que sean compartidos, que los hagan todos o al menos la mayoría. Pero no es sólo por una razón de escalas, de la necesidad de generar un efecto mayor, sino también por la vinculación social que se establece en torno a un bien público como es el medio ambiente. Si alguien no lo hace, no contribuye, recibe la misma recompensa, o prácticamente la misma, que si hubiera hecho lo que debe. Lo que hagan los demás no es indiferente para la percepción y valoración de mi propio comportamiento. La relación entre el coste y el beneficio obtenido por adoptar comportamientos responsables con el medio ambiente, revela un juego descompensado, en el que no hay una compensación suficiente: la recompensa obtenida por el que cumple es la misma que la que obtiene quien incumple.

Normalmente, este tipo de cálculos no se admiten ni se presentan como propios, sino que se atribuyen a otros o se plantean en términos impersonales. Como señalábamos antes, la utilidad social de los comportamientos respetuosos con el medio ambiente, el bien social compartido que se obtiene por su realización bajo la forma de conservación del medio ambiente, no es la única razón ni la más importante para realizarlos. El deber moral que, como vimos, aparece como razón superior para adoptar estos comportamientos, hacer lo que se debe hacer, funciona de esta manera como un factor que impide percibirlos en términos de relación entre coste y beneficio, o al menos impide considerarlos así exclusivamente. Aunque el beneficio obtenido no se corresponda con el coste realizado y aunque esta relación entre coste y beneficio sea mucho más ventajosa para quienes incumplen, esto no justifica que no se haga lo que se debe hacer. $\mathrm{O}$, al menos, no permite utilizar este tipo de argumentos directamente en un contexto de grupo de discusión.

M: Mientras te estás enjabonando no dejar que caiga el agua, cierras, te enjabonas y vuelves a...

M: Yo creo que eso no lo hace la mayoría de la gente.

M: No, no.

M: Yo creo que no tenemos... como algo..., que si para ti fuera un valor, a lo mejor lo harías, ¿sabes? Aunque los demás no lo hagan.

(Grupo de discusión número 3. Entre 18 y 24 años, con estudios superiores)

No obstante, que los demás no cumplan con sus deberes medioambientales tiene también un efecto de desmoralización sobre la propia conducta. La descompensación percibida entre el coste requerido y el beneficio obtenido por quienes cumplen y por quienes incumplen, puede llegar incluso a cuestionar o minimizar la dimensión de estos comportamientos como deber moral. 
Así, se puede llegar a hacer cosas que se creen que no se deben hacer, si se percibe una compensación diferencial respecto de quienes no hacen lo que deben.

M: Mientras que tú te duchas, la gente ésta te está diciendo a ti, los responsables te están diciendo a ti que tú te duches y no te bañes, y ellos están con sus jacuzzi en su casa bañándose... [Risas.] Yo en mi casa sí le lleno a mis hijos la bañera. ¿Lo hacen los demás y mis hijos no se van a bañar?

(Grupo de discusión número 2. Entre 25 y 40 años, sin estudios superiores)

En los grupos, se formulan dos tipos de propuestas para compensar la relación entre los costes y los beneficios percibidos en relación con la realización de los comportamientos respetuosos con el medio ambiente. Se trataría o bien de reducir los costes asociados a estos comportamientos o bien de penalizar los incumplimientos, aumentando así su coste. En cuanto al primer mecanismo, reducir los costes de los comportamientos respetuosos con el medio ambiente, se formulan en los grupos diversas demandas tendentes a facilitar su realización. Se trata, por tanto, de un mecanismo de compensación positiva tendente a incentivar los comportamientos correctos haciéndolos menos costosos para quienes los realizan.

M: Es que es una tarea... Alguna vez lo he hecho lo de las pilas, porque mi marido ha cogido una bolsita y digo: «i $\mathrm{Uy}_{y}$, las pilas, voy a ir a la droguería!». Pero siempre no, siempre no.

$\mathrm{H}$ : Es que debería, yo creo por parte de la Administración, debería facilitar o incentivarte de alguna manera, no te digo ya que te den dinero o algo, sino, no sé, de alguna manera que te incentiven a...

(Grupo de discusión número 2. Entre 25 y 40 años, sin estudios superiores)

Pero quizás el mecanismo de compensación que con mayor frecuencia y fuerza se demanda en los grupos sea el establecimiento de mayores sanciones a los comportamientos poco respetuosos con el medio ambiente. Así, la penalización de los comportamientos irresponsables e irrespetuosos con el medio ambiente se plantea de manera recurrente en todos los grupos de discusión. Se trataría, por tanto, de aumentar el coste asociado a la no-realización de determinados comportamientos como un modo de compensar la relación entre costes y beneficios en función de que se realicen o no se realicen. Por supuesto, este razonamiento no se aplica en primera persona. No se trata de que yo haga lo que sé que debo hacer sólo para evitar la sanción asociada al eventual incumplimiento. Son los demás los que tienen que ver penalizados sus incumplimientos para que mis comportamientos respetuosos tengan sentido. El endurecimiento de las sanciones se aplicaría principalmente a los demás.

M: Si pusieran algo que te duela, tú dices... En lo de los perros, lo de los perros, por ahí, se están contratando detectives para ir a ver si caga el perro porque te multan.

M: Pero eso realmente me da pena, que nosotros nos tengamos que enterar a base de palos. ¿No lo sabes ya? 
M: Pero creo que es la única forma que se están enterando.

(Grupo de discusión número 4. Entre 18 y 24 años, sin estudios superiores)

Este otro generalizado que incumple adquiere, no obstante, concreción dentro de los discursos de los grupos en las empresas contaminantes. En este sentido, se percibe una impunidad respecto de las prácticas contaminantes en la industria y la agricultura. Se considera, en este sentido, que las sanciones son insuficientes, sale más rentable pagar la multa, o bien que no son aplicadas con el suficiente rigor, no se vigila su cumplimiento. Para ser efectivas, las sanciones tendrían que suponer un coste al menos igual al que conlleva el adoptar las medidas correctoras o los comportamientos respetuosos con el medio ambiente. Sólo de esta manera se compensaría la relación entre coste y beneficio, entendido, en este sentido, como evitación de la sanción por el incumplimiento de las normas.

M: Yo pienso que debería haber medidas más fuertes, castigos más fuertes. Ya no sólo a nosotros, sino a la hora, por ejemplo, de construir, a la gente le da igual el sitio, las fábricas. Porque ellos, por ejemplo, sí lo pueden pagar. Ya que a nosotros no nos lo van a hacer porque no nos lo van a hacer y aparte que, entre comillas, sería una putada. Ellos deberían de tener medidas mucho más fuertes, muchísimo más fuertes.

(Grupo de discusión número 4. Entre 18 y 24 años, sin estudios superiores)

En los grupos, se expresa una opinión dominante que defiende la necesidad de endurecer las normas medioambientales y de obligar a su cumplimiento estricto. No se entiende que si algo es bueno para todos, no sea obligatorio. Sin embargo, esta postura punitiva encuentra también su contrapunto en las disculpas. Normalmente, estas disculpas se refieren a las otras razones para incumplir, total o parcialmente, los deberes medioambientales: el desconocimiento, la falta de sensibilización o la incompatibilidad con los modos de vida imperantes.

$\mathrm{H}$ : Se podría pensar a lo mejor en lo que estabais hablando antes, en poner una multa, que la autoridad competente pues le pusiese una denuncia. Pero sin haber información, la suficiente, pues gente mayor, que hay mucha gente en este país no sabe ni... no entiende, ni sabe que las pilas se reciclan siquiera. Y una vez que existiese eso, entonces, pues, ya se podría llegar a pensar en denunciar a la gente.

(Grupo de discusión número 2. Entre 25 y 40 años, sin estudios superiores)

\section{Conclusiones}

1. Los comportamientos respetuosos con el medio ambiente son considerados fundamentalmente como un deber moral: son cosas que hay que hacer porque están bien, porque son lo correcto. Junto a esta motivación moral, aparecen otras dos, a saber, la utilidad social de estos comportamientos, en 
Cuadro 2. Razones para no realizar comportamientos respetuosos con el medio ambiente.

\begin{tabular}{|c|c|c|c|}
\hline Razón & $\begin{array}{l}\text { Factores } \\
\text { motivantes }\end{array}$ & $\begin{array}{l}\text { Factores } \\
\text { intervinientes }\end{array}$ & $\begin{array}{l}\text { Consecuencias sobre } \\
\text { el comportamiento }\end{array}$ \\
\hline Desconocimiento & $\begin{array}{l}\text { - Información } \\
\text { inadecuada } \\
\text { o insuficiente. } \\
\text { - Educación } \\
\text { medioambiental } \\
\text { recibida. }\end{array}$ & $\begin{array}{l}\text { - Carácter complejo } \\
\text { de los comportamientos } \\
\text { requeridos. } \\
\text { - Exigencias percibidas } \\
\text { como crecientes. }\end{array}$ & $\begin{array}{l}\text { - Desconocimiento } \\
\text { o desconsideración } \\
\text { de lo que se debe } \\
\text { hacer. }\end{array}$ \\
\hline Desconfianza & $\begin{array}{l}\text { - Inutilidad percibida } \\
\text { del propio } \\
\text { comportamiento. } \\
\text { - Escasa fiabilidad } \\
\text { concedida a la } \\
\text { información recibida. }\end{array}$ & $\begin{array}{l}\text { - Gestión pública de los } \\
\text { resultados de los } \\
\text { comportamientos } \\
\text { ciudadanos. }\end{array}$ & $\begin{array}{l}\text { - Minimización de la } \\
\text { importancia de los } \\
\text { propios } \\
\text { comportamientos. }\end{array}$ \\
\hline $\begin{array}{l}\text { Relativización } \\
\text { de las exigencias }\end{array}$ & $\begin{array}{l}\text { - Culpabilización } \\
\text { (percibida) del } \\
\text { ciudadano. } \\
\text { - Percepción de las } \\
\text { exigencias como } \\
\text { exageradas. }\end{array}$ & $\begin{array}{l}\text { - Contradicción percibida } \\
\text { entre los valores } \\
\text { medioambientales y los } \\
\text { valores de la sociedad } \\
\text { de consumo, en particular } \\
\text { el valor de la comodidad. }\end{array}$ & $\begin{array}{l}\text { Relativización de las } \\
\text { exigencias: relajación } \\
\text { y discrecionalidad de } \\
\text { las exigencias } \\
\text { asumidas. }\end{array}$ \\
\hline $\begin{array}{l}\text { Ausencia } \\
\text { de compensaciones }\end{array}$ & $\begin{array}{l}\text { Descompensación } \\
\text { entre el coste y el } \\
\text { beneficio percibido, } \\
\text { derivado de la realización } \\
\text { o no de los } \\
\text { comportamientos } \\
\text { respetuosos con el medio } \\
\text { ambiente. }\end{array}$ & $\begin{array}{l}\text { - Ayudas y sanciones } \\
\text { percibidas. }\end{array}$ & $\begin{array}{l}\text { - Desmoralización: } \\
\text { incumplimientos } \\
\text { aunque se reconozca } \\
\text { que está mal. }\end{array}$ \\
\hline
\end{tabular}

particular, su contribución a la conservación del medio ambiente, y los beneficios particulares, para la salud o para la economía, principalmente, derivados de muchos de estos comportamientos. Estas motivaciones no son incompatibles entre sí, sino que más bien se suman.

2. Los comportamientos respetuosos con el medio ambiente son percibidos como costosos. Este coste puede ser una molestia, un esfuerzo, una renuncia o un desembolso económico. Este coste asociado a los comportamientos respetuosos con el medio ambiente no es en sí mismo un factor que desmotive su realización. Por el contrario, el coste se considera como algo consustancial a los comportamientos respetuosos con el medio ambiente: un comportamiento que no contamine pero que no suponga un coste no es considerado, en este sentido, un comportamiento medioambiental, sino simplemente no-contaminante. No obstante, con frecuencia se percibe el coste que suponen determinados comportamientos como excesivo o exa- 
gerado. En estos casos, el coste sí puede ser un factor que condicione la realización del comportamiento medioambiental, si bien no como impedimento, sino como limitación del grado de exigencia.

3. En los discursos analizados, aparecen cuatro razones fundamentales para la no-realización de comportamientos respetuosos con el medio ambiente: el desconocimiento, la desconfianza, la limitación de las exigencias y la ausencia de compensaciones. El desconocimiento se refiere tanto a las carencias de información como a la falta de sensibilidad hacia los problemas medioambientales. La desconfianza alude generalmente a las dudas en torno a la repercusión o utilidad de los comportamientos requeridos. En este sentido, suele centrarse en la gestión pública de los problemas medioambientales. La limitación de las exigencias tiene que ver con la percepción de una excesiva responsabilización de los ciudadanos y del excesivo coste de los comportamientos respetuosos con el medio ambiente, lo que lleva a asumirlos de manera limitada, y ello introduce elementos de relajación y discrecionalidad en su realización. Por último, la ausencia de compensaciones se refiere al cálculo entre los costes y los beneficios de la realización o no de comportamientos respetuosos con el medio ambiente. Básicamente, esta actitud negativa se deriva de constatar que el beneficio obtenido no se corresponde con el coste realizado, lo que supondría un agravio comparativo para quienes lo realizan. Aunque muchas de las sugerencias formuladas en los grupos tienen que ver con la reducción de costes, con facilitar los comportamientos responsables, en la mayoría de los casos se demanda un endurecimiento de las sanciones ante los incumplimientos como el modo de compensar negativamente a quienes no las adoptan.

4. La conciencia medioambiental, vale decir el conocimiento y la sensibilización acerca de los problemas medioambientales, se ha generalizado socialmente, al menos en el contexto y entre los grupos sociales en los que se ha centrado esta investigación. Pero también se ha generalizado una mentalidad que podemos denominar como «de responsabilidad limitada» respecto del medio ambiente. Esta mentalidad explica, en buena medida, la nocorrespondencia o inconsistencia entre la conciencia medioambiental y los comportamientos relacionados con el medio ambiente. En este sentido, la conciencia medioambiental se muestra como una condición insuficiente para el mantenimiento de comportamientos respetuosos con el medio ambiente. Dicho de otro modo, se puede tener una alta conciencia medioambiental y, sin embargo, no mantener comportamientos respetuosos con el medio ambiente, o sólo mantenerlos parcialmente, en la medida en que se asuman sólo de manera limitada los requerimientos que tales comportamientos tienen para la vida cotidiana.

5. El binomio información-concienciación no es automático. No siempre una mayor información se traduciría en una mayor concienciación, por varias razones. Por un lado, en ocasiones se desconfía de la información disponible sobre el medio ambiente, en concreto de la información comercial o de lo que se considera como tal, lo que limitaría decisivamente su eficacia. El 
desgaste del término ecológico, aplicado a productos de consumo, es un ejemplo de esta desconfianza. Por otro lado, el exceso de información tiene un efecto de saturación que puede llevar a su rechazo. Éste es el caso de la percepción de los requerimientos medioambientales como exagerados e, incluso, ilimitados. Por último, el carácter complejo de la información suministrada sobre los problemas medioambientales puede llevar a una recepción desigual por parte de la ciudadanía. Ésta puede ser una de las causas de la desigual "concienciación» en función del nivel de estudios, entendida como nivel de conocimiento de los problemas medioambientales.

6. En la población en la que se ha centrado este estudio, no se han detectado diferentes mentalidades medioambientales. Los andaluces entre $18 \mathrm{y}$ 40 años residentes en zonas urbanas presentan discursos sobre el medio ambiente muy similares, que reflejan una mentalidad medioambiental generalizada. Esto no quiere decir que no se encuentren diferencias discursivas en función de la edad o del nivel de estudios, pero se trata de diferencias de matiz que no hacen pensar en diferentes mentalidades. Sería preciso ampliar el estudio a otros contextos y a otros colectivos para explorar otras mentalidades medioambientales y su incidencia en los comportamientos relacionados.

\section{Bibliografía}

ADAms, H.; Props, J. (ed.) (2001). Social discourse and environmental policy: An application of $Q$ methodology. Northampton: Edward Elgar Pub.

Alonso, L.E. (1998). La mirada cualitativa en sociología. Madrid: Fundamentos. BRAND, K.-W. (1997). «Conciencia y comportamientos medioambientales: estilos de vida más "verdes"». En: REDCLIFT, M.; WOODGATE, G. Sociología del medio ambiente: Una perspectiva internacional. Madrid: Mc Graw-Hill.

Brand, K.; POFERL, W.A.; SCHILling, K. (1998). «Umweltmentalitäten. Wie wir die Umwelt-thematik in unser Alltagsleben integrieren». En: HAAN, G.; KuCKARTZ, U. (ed.). Umweltbildung und Umweltbewusstsein. Opladen: Leske y Budrich, p. 39-68.

Brand, K-W.; C. Fischer; Hofmann, M. (2003). «Lebensstile, Unweltmentalitaeten und Unweltverhalten in Ostdeutschland». UFZ, Bericht, 11/2003. Unweltforschungszentrum, Halle.

CALLEJO, J. (2001). El grupo de discusión: Introducción a una práctica de investigación. Barcelona: Ariel.

DARIER, E. (1999). Discourses of the environment. Oxford: Blackwell.

DunlaP, R.E.; VAN LieRE, K. (1983). «Commitment to the Dominant Social Paradigm and Concern for environmental Quality». Social Science Quarterly, 9.

DryzeK, J. (1997). The Politics of the Earth: Environmental Discourses. Nueva York: Oxford University Press.

EDER, K. (1992). Framing and communicating environmental issues: A discourse analysis of environmentalist. Florencia: European University Institute.

Eder, K.; Kousis, M. (eds). (2000). Environmental Politics in Southern Europe. Dordrecht: Kluwer. 
FARR, R. M.; MOSCOVICI, S. (eds.). (1984). Social Representations. Cambridge, Inglaterra: Cambridge University Press.

Fuhrer, U.; Keiser, F. G.; Seiter, I.; Maggi, M. (1995). «Environmental Concern as a Function of Social Representation». En: FUHRER, U. (ed.). Ökologische Handeln als sozialer Proze,. Basle: Birkhäuser.

GÓmeZ, C.; NoyA, J.; PANIAGUA, A. (1999). Actitudes y comportamientos hacia el medio ambiente en España. Madrid: CIS.

- (1999b). "La inconsistencia de las actitudes hacia el medio ambiente en España». En: PARDO, M. (coord.). Sociología y medio ambiente: Estado de la cuestión. Madrid: Fundación Fernando de los Ríos. Universidad Pública de Navarra.

HAJER, M. A. (1995). The politics of environmental discourse: Ecological modernization and the policy process. Oxford: Clarendon Press.

IBÁÑEZ, J. (1989). "Cómo se realiza una investigación mediante grupos de discusión». En: Alvira, F.; IbÁÑEZ, J.; GarCía FERRANDO, M. El análisis de la realidad social: Métodos y técnicas de investigación. Madrid: Alianza Editorial.

Instituto de Estudios Sociales de Andalucía (1996). Percepción de los problemas medioambientales por la población andaluza. Córdoba: IESA.

JONES, R.E.; DUnLAP, R.E. (1992). "The Social Basis of Environmental Concern: Have they Changed Over Time?». Rural Sociology, 57 (1).

KuCKARTZ, U. (1995). "Umweltwissen, Umwelbewu,tsein, Umweltverhalten. Der Stand der Umweltbewu,tseinsforschung». En: GERD DE HAAN (ed.). Umwelbewu,tsein und Massenmedien;: Perspektiven Ökologischer Kommunikation. Berlín: Akademie Verlag.

Martín Crespo, T. (1999). «Percepción social del medio ambiente en España. Perspectiva del movimiento ecologista». En: PARDO, M. (coord.). Sociología y medio ambiente: Estado de la cuestión. Madrid: Fundación Fernando de los Ríos. Universidad Pública de Navarra.

NAVARro YÁneZ, C. J. (1998). «Conciencia ambiental y perfil social del ambientalismo». Revista Internacional de Sociología (RIS), n. ${ }^{\circ}$ 19-20.

Olsen, M. E.; LODWICK, D. G.; DunlaP, R. E. (1992). Viewing the World Ecologically. Bolder: Westview.

Poferl, A.; SCHILling, K.; Brand, K-W. (1997). Ökologische frage und aletagshaudelu. Opaladen: Leske y Budrich.

SANTAMARINA, B. (2004). «Entre la consciencia y la inconsciencia medioambiental de los españoles. Paradojas y debilidades». Alicante: Ponencia presentada en el VIII Congreso de la Federación Española de Sociología.

SCHAHN, J.; Holzer, E. (1990). «Studies of Individual Environmental Concern. Role of Knowledge, Gender and Background Variables». Environment and Behavior, 22.

SKrenTNY, J. (1993). "Concern for the Environment: A Cross-National Perspective». International Journal of Public Opinion Research, 5(4).

TÁBARA, J. D. (2001). «La percepción social del medio ambiente. Una revisión de las aportaciones realizadas por la sociología». Revista Internacional de Sociología (RIS), n. ${ }^{\circ} 28$, enero-abril. 